\title{
Relación entre Puntaje de Prueba de Selección Universitaria y Nota Enseñanza Media, y el Rendimiento Académico de la Asignatura de Morfología en Alumnos de Primer Año de Odontología de la Universidad de Los Andes
}

Relationship between University Selection Test Scores, High School Grade Point Average (GPA) and Academic Performance in the Subject of Morphology in Dentistry Freshmen at the Universidad de los Andes

Grob, M.*; Becerra, D.*; Rodriguez, A.."; Cristiane, J."; Ramirez, V." \& Sabag, N.*

GROB, M.; BECERRA, D.; RODRIGUEZ, A.; CRISTIANE, J.; RAMIREZ, V. \& SABAG, N. Relación entre puntaje de prueba de selección universitaria y nota enseñanza media, y el rendimiento académico de la asignatura de morfología en alumnos de primer año de Odontología de la Universidad de los Andes. Int. J. Morphol., 33(2):527-531, 2015.

RESUMEN: El estudio de predictores de rendimiento académico presenta gran relevancia en el área de la salud. El objetivo de este trabajo fue evaluar la relación entre el puntaje de Prueba de Selección Universitaria (PSU) y notas de enseñanza media (puntaje nota de enseñanza media) en la asignatura de Morfología en alumnos de primer año de odontología, en la Universidad de los Andes. Estudio descriptivo con 312 estudiantes admitidos en el primer año de la carrera de odontología de la Universidad de los Andes de Chile, de forma regular y que terminaron el año universitario sin deserción los años 2005 a 2008 y 2010. Las variables estudiadas fueron género, promedio puntaje de notas de enseñanza media, puntaje PSU, tipo de colegio de procedencia según sistema de financiamiento (municipal, particular subvencionado y particular) y nota final de la asignatura de morfología de los alumnos del primer año de estudio, en la carrera de odontología. De los 312 alumnos, la mediana de PSU, notas de enseñanza media y nota de morfología fueron 692,6, 702 y 4,4 respectivamente. Del total de la muestra, 221 alumnos (71\%) correspondieron al sexo femenino. Del total de la muestra, 290 alumnos (93\%) provenían de colegios particulares, 15 alumnos (5\%) de colegios particulares subvencionados y 6 alumnos (2\%) de colegios municipales. La relación entre puntaje nota de enseñanza media y nota de morfología muestra una correlación de 0,35 (Rho de Spearman, valor-p <0,0001), y puntaje de PSU con nota de Morfología una correlación de 0,39 (Rho de Spearman, valor-p <0,0001). Existe asociación entre nota morfología, puntaje de notas de enseñanza media y PSU, de tal manera, que los mejores puntajes de nota de enseñanza media y PSU, muestran mejor rendimiento en la asignatura de morfología.

PALABRAS CLAVE: Criterios de Admisión; Prueba de selección Universitaria; Morfología; Educación.

\section{INTRODUCCIÓN}

El estudio de predictores de rendimiento académico toma relevancia en el área de la salud (Zuñiga et al., 2009; Rodríguez Fontes et al., 2000). Predecir el eventual desempeño académico de los estudiantes de odontología ha sido estudiado con anterioridad, en base a mediciones de admisión (Stacey \& Whittaker, 2005).

Se ha intentado determinar como influyen distintos factores en el desempeño de pregrado, tales como: variables demográficas, estilo de aprendizaje y notas de enseñanza media (Zuñiga, 2009). Los estudios confirman que una medición por si sola como predictor académico es insuficiente, y la literatura respalda que una combinación de variables sería más certero en su predicción, incluyendo una medición estandarizada, evaluación escolar, entrevista y evaluación vocacional (Stacey \& Whittaker).

El sistema actualmente utilizado en Chile para la admisión a las distintas carreras en distintas universidades, es la Prueba de Selección Universitaria (PSU), la cual se comenzó a implementar desde el año 2002 por el organismo estatal: Departamento de Evaluación, Medición y Registro Educacional (DEMRE, 2015) y las notas de enseñanza media.

La PSU corresponde a una prueba de selección múltiple, dividida en tres partes: matemáticas, lenguaje y ciencias sociales o ciencias naturales. Esta última, consiste en una prueba básica común y luego una específica, en donde 
GROB, M.; BECERRA, D.; RODRIGUEZ, A.; CRISTIANE, J.; RAMIREZ, V. \& SABAG, N. Relación entre puntaje de prueba de selección universitaria y nota enseñanza media, y el rendimiento académico de la asignatura de morfología en alumnos de primer año de Odontología de la Universidad de los Andes. Int. J. Morphol., 33(2):527-531, 2015.

el alumno puede elegir la asignatura de química, física o biología, con lo cual se persigue una conexión entre las áreas del conocimiento de ciencias naturales, humanidades y ciencias sociales (DEMRE). Estas pruebas miden la capacidad de razonamiento del alumnado, evaluando así sus habilidades cognitivas, dentro de las categorías: recordar, comprender, aplicar, analizar, evaluar y crear, así como el modo de operar y de analizar problemas (DEMRE).

Por otra parte, la asignatura de morfología, que se imparte en la mayor parte de las escuelas del área de la salud, es la asignatura de mayor dificultad en el primer año y requiere de gran capacidad de observación y memoria por parte del alumno. En la Universidad de los Andes comprende anatomía, histología y embriología.

El presente estudio tiene por objetivo evaluar la relación entre el puntaje de PSU y notas de enseñanza media (puntaje nota de enseñanza media) en la asignatura de morfología en alumnos de primer año de odontología, en la Universidad de los Andes.

\section{MATERIAL Y MÉTODO}

Se llevó a cabo un estudio descriptivo en el cual se analizó la información de 312 estudiantes admitidos en el primer año de la carrera de odontología de la Universidad de los Andes de Chile, de forma regular y que terminaron el año universitario sin deserción los años 2005 a 2008 y 2010. El año 2009 no se incluyó ya que no se contó con todos los datos necesarios.
La información se obtuvo a través de una base de datos anónima entregada por la secretaría de estudio de la Facultad de Odontología de la Universidad de los Andes.

Se analizaron los resultados de las pruebas de ingreso exigidos para postular a la carrera de odontología en la Universidad de los Andes entre 2005 a 2008 y 2010. La Prueba de Selección Universitaria de Lenguaje y Comunicación, la Prueba de Selección Universitaria Matemáticas, la Prueba de Selección Universitaria Ciencias y el puntaje de Selección que se obtiene de la ponderación que aportan las pruebas.

Las variables estudiadas fueron género (variable dicotómica), promedio puntaje de notas de educación media (variable continua), puntaje PSU (variable continua), tipo de colegio de procedencia según sistema de financiamiento (municipal, particular subvencionado y particular; variable nominal) y nota final de la asignatura de morfología de los alumnos del primer año de estudio, en la carrera de odontología (variable continua).

Análisis Estadístico. Las variables continuas se describieron con medidas de tendencia central y dispersión y las variables nominales se describieron con frecuencias absolutas y porcentajes. Se evaluó la correlación entre las variables continuas a través del coeficiente de correlación de Spearman. El procesamiento de la información se realizó a traves del programa STATA versión 12.

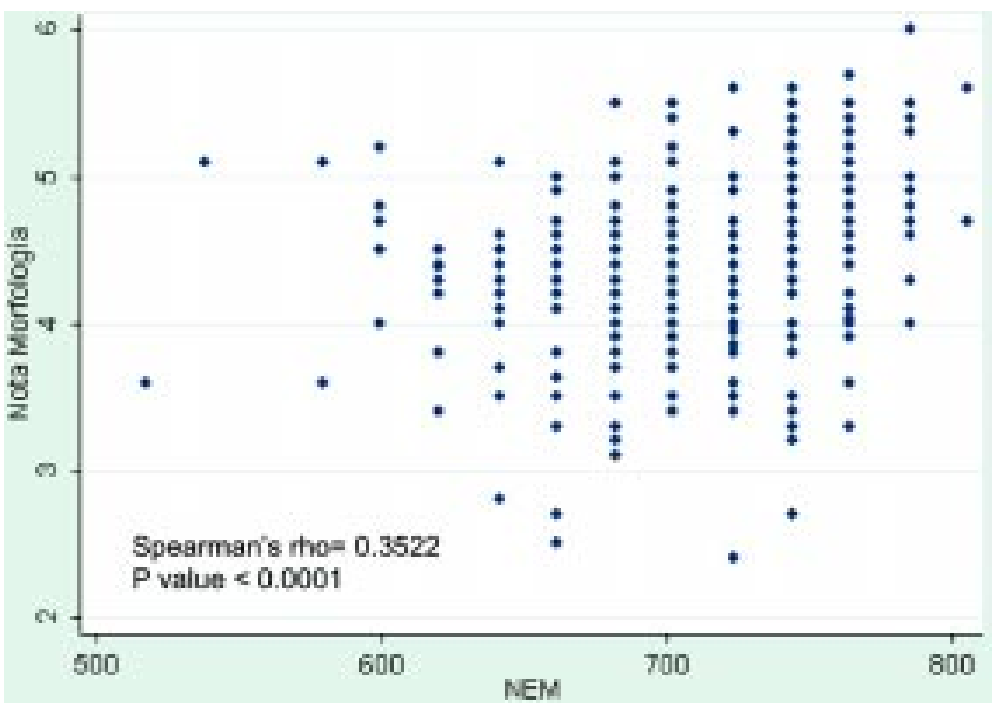

Fig. 1. Gráfico de dispersión: Nota morfología - Puntaje nota de enseñanza media.

\section{RESULTADOS}

El estudio incluyó 312 alumnos, que ingresaron los años 2005 a 2008 y 2010, la mediana de PSU, notas de enseñanza media y nota de morfología fueron 692,6, 702 y 4,4 (escala de 1 a 7 ) respectivamente. Del total de la muestra, 221 alumnos (71\%) correspondieron al género femenino.

La mediana de alumnos por año fue 61. Del total de la muestra, 290 alumnos (93\%) provenía de colegios particulares, 15 alumnos $(5 \%)$ de colegios particulares subvencionados y 6 alumnos (2\%) de colegios municipales.

La relación entre puntaje nota de enseñanza media y nota de morfología muestra una correlación de 0,35 (Rho de Spearman, valor-p <0,0001) (Fig. 1) y puntaje de PSU con nota de morfología una correlación de 


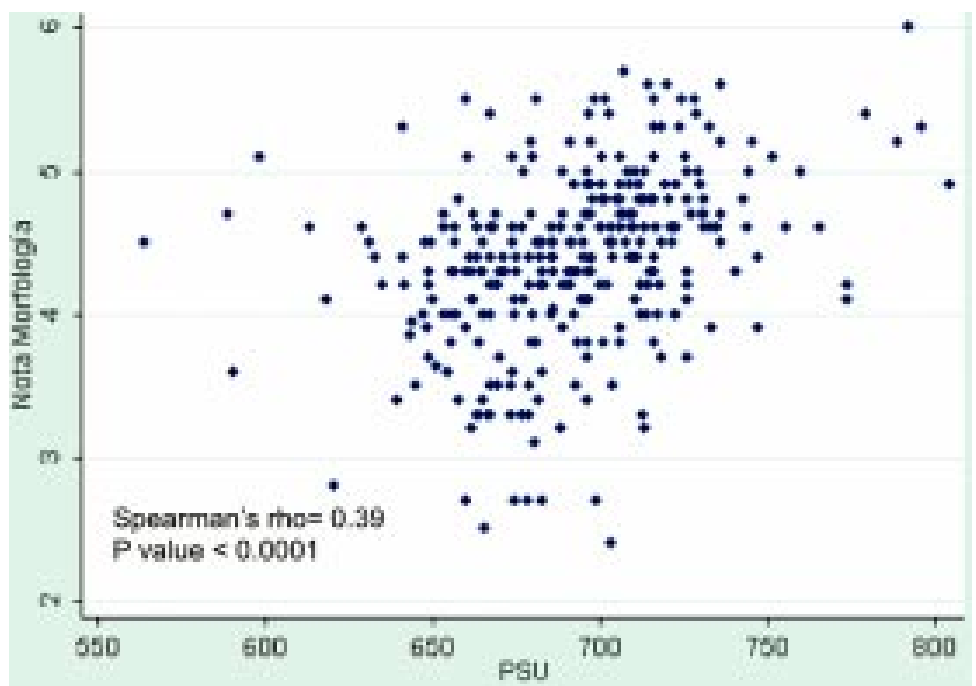

Fig. 2. Gráfico de dispersión: Nota morfología - PSU.

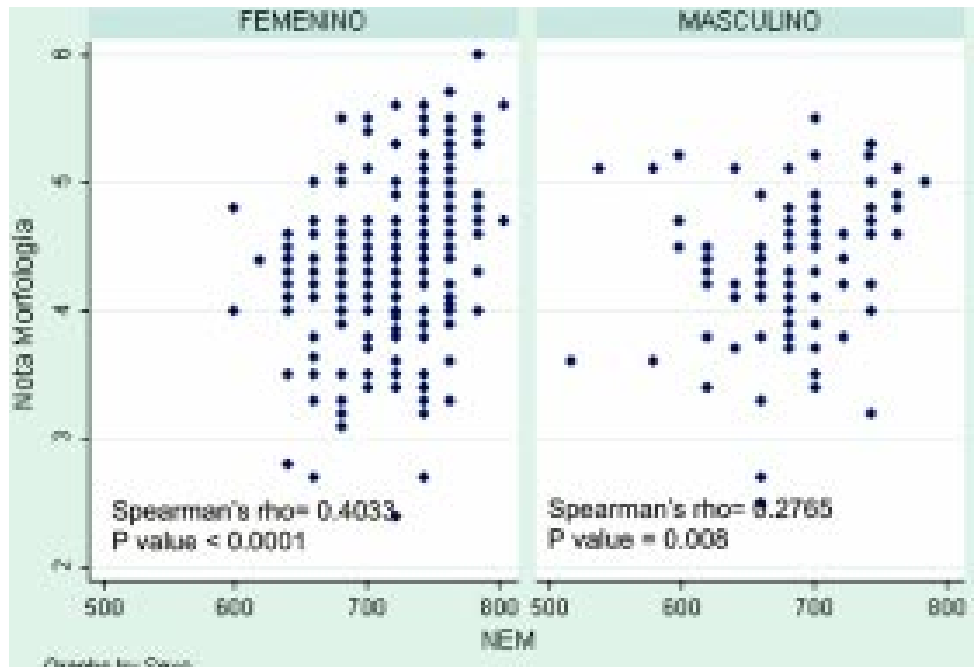

Fig. 3. Gráfico de dispersión según sexo: Nota morfología - Puntaje nota de enseñanza media.

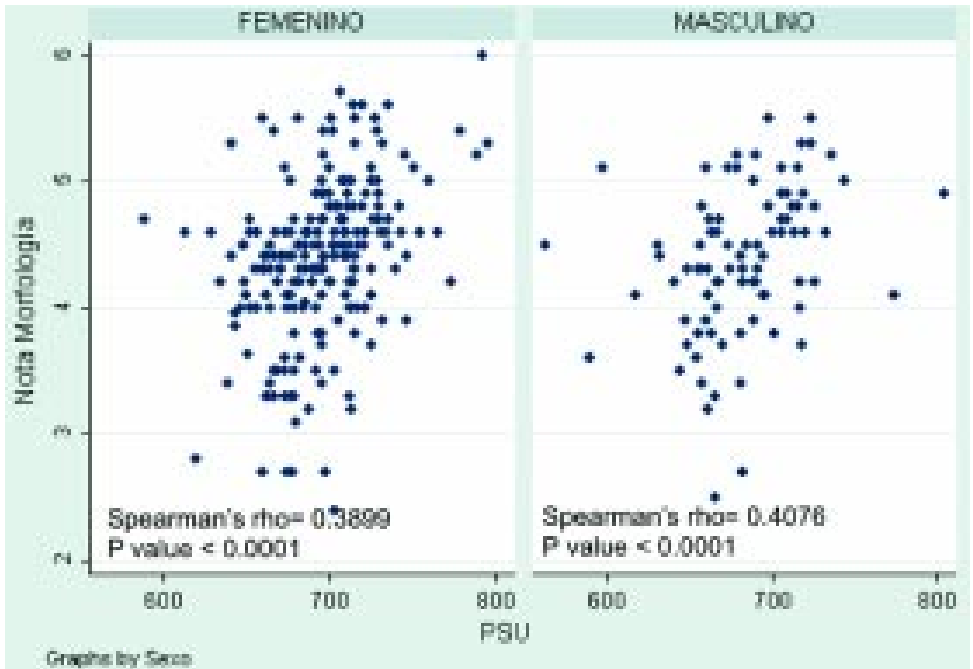

Fig. 4. Gráfico de dispersión según sexo: Nota morfología - PSU.
0,39 (Rho de Spearman, valor-p <0,0001) (Fig. 2).

Las mujeres presentaron una correlación de 0,4 (Rho de Spearman, valor $\mathrm{p}$ $<0,0001)$ para nota de morfología con nota de enseñanza media (Fig. 3) y de 0,38 (Rho de Spearman, valor $\mathrm{p}<0,0001)$ para nota de morfología y puntaje de PSU (Fig. 4).

En los colegios particulares, la correlación para nota morfología con puntaje de nota de enseñanza media fue de 0,35 (Rho de Spearman, valor-p $<0,00001)$, nota morfología con PSU fue de 0,37 (Rho de Spearman, valor-p <0,00001) y PSU con puntaje de notas de enseñanza media de 0,56 (Rho de Spearman, valor - $\mathrm{p}<0,00001)$. En los colegios subvencionados la correlación para nota de morfología con puntaje de notas de enseñanza media fue de 0,32 (Rho de Spearman, valor $-\mathrm{p}=$ 0,2123 ) y nota morfología con PSU fue de 0,35 (Rho de Spearman, valor - $\mathrm{p}=0,1802$ ) y PSU con puntaje de notas de enseñanza media fue de 0,18 (Rho de Spearman, valor - $\mathrm{p}=0,4837$ ). En los colegios municipales, la correlación para nota morfología con puntaje de notas de enseñanza media fue de 0,25 (Rho de Spearman, valor - $\mathrm{p}=0,5852$ ), nota morfología con puntaje PSU fue de 0,14 (Rho de Spearman, valor $-\mathrm{p}=$ $0,7599)$ y para PSU con puntaje de notas de enseñanza media fue de 0,95 (Rho de Spearman, valor $-\mathrm{p}=0,0008$ ).

Respecto al año de ingreso a la carrera, el puntaje de notas de enseñanza media y nota de morfología mostró una correlación para el año 2005 de 0,46 (Rho de Spearman, valor - $\mathrm{p}=0,0002$ ), para el año 2006 de 0,45 (Rho de Spearman, valor $-\mathrm{p}=0,0003$ ), para el año 2007 de 0,58 (Rho de Spearman, valor - $\mathrm{p}<0,00001$ ), para el año 2008 de 0,18 (Rho de Spearman, valor $-\mathrm{p}=0,1392$ ) y para el año 2010 de 0,22 (Rho de Spearman, valor $-\mathrm{p}=0,0535)$. Notas Morfología - PSU mostraron una correlación para el año 2005 de 0,59 (Rho de Spearman, valor $-p$ $<0,00001$ ), para el año 2006 de 0,52 (Rho de Spearman, valor $-\mathrm{p}=0,0000$ ), para el año 2007 de 0,45 (Rho de Spearman, valor $-\mathrm{p}$ $=0,001)$, para el año 2008 de 0,04 (Rho de Spearman, valor $-\mathrm{p}=0,7162)$ y para el año 2010 de 0,51 (Rho de Spearman, valor $\mathrm{p}<0,00001)$. 


\section{DISCUSIÓN}

De acuerdo a nuestros resultados, se observó que existe correlación entre nota morfología, puntaje PSU y puntaje nota de enseñanza media. A mayor puntaje PSU y puntaje nota de enseñanza media, mejor rendimiento académico en morfología.

Al analizar la nota morfología, puntaje de notas de enseñanza media y PSU observamos que existe asociación entre las variables, de tal manera, que los mejores resultados de puntaje nota de enseñanza media y PSU, muestran mejor rendimiento en la asignatura de morfología. Estudios anteriores relatan que puntaje nota de enseñanza media es uno de los puntajes más predictores en el rendimiento de los alumnos de odontología (Sandow et al., 2002; Wilkinson et al., 2008; Bastias et al., 2000), sin embargo otros autores (Curtis et al., 2007) postulan que los criterios de admisión son débiles en términos de predecir el éxito en el primer año de la carrera de odontología, a lo que posteriormente se agregó el puntaje de prueba de selección universitaria para complementar la información.

En general, los alumnos con mejores puntajes de notas de enseñanza media, tienen mejores hábitos de estudio, lo que explicaría esta asociación. La mayor correlación se observa entre PSU y puntajes de notas de enseñanza media, lo que es esperable, por ser variables requisitos para el ingreso a odontología.

Dentro de las características demográficas, el género tiene una relación importante con el desempeño en la asignatura de morfología el primer año de odontología. Consistente con estudios anteriores (Kim \& Lee, 2007), las mujeres presentan una mejor correlación entre nota morfología, puntaje de notas de enseñanza media y PSU que los hombres.

Las mejores notas de los alumnos de colegios particulares muestran mejor y mayor asociación con mejores notas de morfología, ya que existe correlación significativa para nota morfología con puntaje de notas de enseñanza media, nota morfología con PSU y PSU con puntajes de notas de enseñanza media.

Respecto a los colegios subvencionados, no hay correlación significativa para nota de morfología con PSU ni PSU con puntaje nota de enseñanza media. Los colegios municipales por otro lado, no mostraron correlación para nota morfología con puntaje nota de enseñanza media, nota morfología - PSU, pero sí para PSU con puntaje nota de enseñanza media.
Esta mayor correlación demostrada para los colegios municipales entre puntaje nota de enseñanza media y PSU, se puede deber a que los alumnos que entran a la carrera de odontología, y que provienen de colegios municipales, son escogidos precisamente por sus buenas calificaciones de puntaje nota de enseñanza media y PSU.

Respecto al año de ingreso a la carrera de odontología, se observa una menor correlación entre nota morfología - PSU.

Una de las limitaciones del estudio, radica en que la muestra es solo de los alumnos de la Univeridad de los Andes. Replicar este estudio en diferentes universidades de Chile otorgará una muestra mayor para determinar para determinar si estos resultados pueden generalizarse a lo largo de la población demográfica del país. Por otro lado, la relación entre diversos puntajes de admisión y el desempeño de morfología, podría verse alterarada por otras variables no consideradas en este estudio.

La contribución de este trabajo, frente a los criterios de admisión y el desempeño de cada asignatura, podría ser evaluada a través de un estudio longitudinal, que determine si la correlación se mantiene en cursos clínicos superiores.

Este estudio nos permite tener una visión sobre los criterios de selección universitaria (puntaje nota de enseñanza media y PSU) y si son capaces de determinar el rendimiento que tendrán los alumnos en la asignatura de morfología, ramo de mayor dificultad durante el primer año.

El presente análisis podría ayudar a las facultades de Odontología de Chile a entender como las características demográficas de los estudiantes y sus puntajes de admisión pueden ser interpretados y correlacionados para predecir su rendimiento académico durante el primer año universitario.

\section{AGRADECIMIENTOS}

Se agradece la colaboración de Victoria Riumallo por su aporte en la recolección de la información y a Valeria Ramirez por su gran aporte en la parte de análisis estadístico. 
GROB, M.; BECERRA, D.; RODRIGUEZ, A.; CRISTIANE, J.; RAMIREZ, V. \& SABAG, N. Relación entre puntaje de prueba de selección universitaria y nota enseñanza media, y el rendimiento académico de la asignatura de morfología en alumnos de primer año de Odontología de la Universidad de los Andes. Int. J. Morphol., 33(2):527-531, 2015.

GROB, M.; BECERRA, D.; RODRIGUEZ, A.; CRISTIANE, J.; RAMIREZ, V. \& SABAG, N. Relationship between test score and grade selection of university and high school, and academic performance in the subject of morphology freshmen of dentistry, at university of the Andes. Int. J. Morphol., 33(2):527-531, 2015.

SUMMARY: The study of predictors of academic performance is of great importance in the area of health. The objective of this study was to assess the relationship between PSU (University selection admission scores) and Secondary Education (High school grade point average score) in the freshmen morphology course in dentistry, at the Universidad de los Andes. A descriptive study of 312 students admitted to the first year of dental school at the Universidad de los Andes in Chile, on a regular basis and who have completed the academic year without dropping out, the years 2005-2008 and 2010. The variables studied were sex, grade point average in high school, PSU score, type of school of origin as financing system (municipal, private subsidized and private) and final grade received in morphology for dentistry students during their freshmen year. Of the 312 students, the median PSU, high school grades and morphology were as follows: 692.6, grade 702, and 4.4 respectively. Of the total sample, 221 students (71\%) were female. Of the total sample, 290 students $(93 \%)$ had attended private schools, 15 students (5\%) private subsidized schools and 6 students (2\%) municipal schools. The relationship between scores and high school grade point average and grade attained in the morphology course, shows a correlation of 0.35 (Spearman rho, p-value <0.0001) and PSU score with the morphology course grade, a correlation of 0.39 (Spearman rho, p-value $<0.0001$ ). There is a relationship between morphology grade scores, high school grades and university selection test scores, indicating that the best high school grades and university selection scores show better student performance in the subject of morphology.

\section{KEY WORDS: Admission criteria; University selection test; Morphology; Education.}

\section{REFERENCIAS BIBLIOGRÁFICAS}

Bastias, G.; Villarroel, L.; Zuñiga, D.; Marshall, G.; Velasco, N. \& Mena, B. Academic performance of medical students: a predictable result? Rev. Med. Chil., 128(6):671-8, 2000.

Curtis, D. A.; Lind, S. L.; Plesh, O. \& Finzen, F. C. Correlation of admissions criteria with academic performance in dental students. J. Dent. Educ., 71(10):1314-21, 2007.

Departamento de Evaluación, Medición y Regisgro Educacional (DEMRE). PSU: Prueba de Selecciónn Universitaria. Santiago de Chile, Universidad de Chile, 2015. Disponible en: http://www.portaldemre.demre.cl

Kim, M. \& Lee, J. I. Variables predicting students' first semester achievement in a graduate-entry dental school in Korea. $J$. Dent. Educ., 71(4):550-6, 2007.

Rodríguez Fontes, R.; Bacallao Gallestey, J.; Díaz Rodríguez, P. A. \& Morejón Lazo, M. Valor predictivo de algunos criterios de selección para el ingreso a la carrera de medicina. Educ. Med. Super., 14(1):17-25, 2000.

Sandow, P. L.; Jones, A. C.; Peek, C. W.; Courts, F. J. \& Watson, R. E. Correlation of admission criteria with dental school performance and attrition. J. Dent. Educ., 66(3):385-92, 2002.

Stacey, D. G. \& Whittaker, J. M. Predicting academic performance and clinical competency for international dental students: seeking the most efficient and effective measures. J. Dent. Educ., 69(2):270-80, 2005.
Zúniga, D.; Mena, B.; Oliva, R.; Pedrals, N.; Padilla, O. \& Bitran, M. Modeling the academic performance of medical students in basic sciences and pre-clinical courses: a longitudinal study. Rev. Med. Chil., 137(10):1291-300, 2009.

Wilkinson, D.; Zhang, J.; Byrne, G. J.; Luke, H.; Osolins, I. Z.; Parker, M. H. \& Peterson, R. F. Medical school selection criteria and the prediction of academic performance: Evidence leading to change in policy and practice at the University of Queensland. Med. J. Aust., 188(6):349-54, 2008.

\section{Dirección para Correspondencia: \\ Melisa Grob \\ Unidad de Morfología \\ Facultad de Odontología \\ Universidad de los Andes \\ Santiago \\ CHILE}

Email:melisagrob@gmail.com

Recibido: 23-11-2014

Aceptado: 19-03-2015 